\title{
Low-Dimensional Inorganic Nanofunctional Materials: Design, Assembly, and Application for Chemical Engineering (I)
}

\author{
Lan Xiang, ${ }^{1}$ Guo Gao, ${ }^{2}$ Yunpeng Yin, ${ }^{3}$ Huijun Wu, ${ }^{4}$ Yongcheng Jin, ${ }^{5}$ and Wancheng $\mathrm{Zhu}^{6}$ \\ ${ }^{1}$ Department of Chemical Engineering, Tsinghua University, Beijing 100084, China \\ ${ }^{2}$ Department of Bio-Nano-Science and Engineering, Shanghai Jiao Tong University, Shanghai 200240, China \\ ${ }^{3}$ IBM Albany NanoTech Research and Development Center, Albany, NY 12309, USA \\ ${ }^{4}$ College of Civil Engineering, Guangzhou University, Guangzhou 510006, China \\ ${ }^{5}$ Department of Applied Chemistry, Tokyo Metropolitan University, Tokyo 00813, Japan \\ ${ }^{6}$ Department of Chemical Engineering, Qufu Normal University, Qufu 273165, China
}

Correspondence should be addressed to Lan Xiang; xianglan@mail.tsinghua.edu.cn

Received 25 August 2013; Accepted 25 August 2013

Copyright (C) 2013 Lan Xiang et al. This is an open access article distributed under the Creative Commons Attribution License, which permits unrestricted use, distribution, and reproduction in any medium, provided the original work is properly cited.

Synthesis of low-dimensional inorganic nanofunctional materials is an increasingly important subject for chemical engineering researchers owing to their intriguing properties and wide applications in many chemical engineering fields such as ceramics, catalyst, and composite. Great progress was achieved in both theoretical and technical aspects for lowdimensional inorganic nanofunctional materials with desired composition, morphology, structure, and even facets. Thus, fabricating the low-dimensional inorganic nanofunctional materials, revealing the size-, shape-, composition-, and surface-dependent properties, and uncovering the potential applications in chemical engineering fields have turned to be an urgent and booming issue.

This special issue focuses on the development of new theories and technologies for the synthesis, characterization, and application of low-dimensional inorganic nanofunctional materials, especially those with potential applications in chemical engineering fields. A total of 13 research articles reporting on the design and assembly of various shaped lowdimensional nanomaterials including nanoparticles (B. Yu and L. Xiang, Y. Zhang, and N. Zheng et al.), nanosheets (J. Liu et al., B. Yu, and L. Xiang), nanofibers (H. Wu et al.), as well as micro-nano spheres and whiskers (L. Yang and L. Xiang, S. C. Hou and L. Xiang, K. Luo et al., Y. Zhang, and S. Zhang et al.) are presented in the special issue. The articles cover diversified object materials such as $\mathrm{Cu}$ (Y. Zhang and
N. Zheng et al.), $\mathrm{SiO}_{2}$ (H. Wu et al.), $\mathrm{TiO}_{2}$ (Y. Zhang and S. Zhang et al.), $\mathrm{ZnO}$ (L. Yang and L. Xiang), $\mathrm{Al}(\mathrm{OH})_{3}$ (B. Yu and L. Xiang), $\mathrm{CaSO}_{4}$ (S. C. Hou and L. Xiang and K. Luo et al.), graphene (J. Liu et al.), Sn/Ni (Y. Jin et al.), and Fe/Mn nanocomposites (W. Weilong and F. Xiaobo). The traditional fabrication processes such as precipitation (B. Yu and L. Xiang and L. Yang and L. Xiang), phase separation (L. Ge et al.), hydrothermal conversion ( $\mathrm{K}$. Luo et al. and J. Wang et al.), solvothermal reaction (Y. Zhang and S. Zhang et al.), and sol-gel process $(\mathrm{H}$. Wu et al. and Y. Zhang and N. Zheng et al.) have been extensively presented in the synthesis of the nanomaterials, less common but promising methods such as electrospinning $(\mathrm{H}$. Wu et al.), colloidal crystal templating and electroplating process (Y. Jin et al.), and grinding (W. Weilong and F. Xiaobo), are also introduced.

The application prospects relevant to chemical engineering in these articles range from reinforcing materials (S. C. Hou and L. Xiang and K. Luo et al.) to thermal insulations (H. Wu et al.) and anti-icing (L. Ge et al.), from nanofiltration separation ( $\mathrm{Z}$. Chen et al.) to heavy metal removal (W. Weilong and F. Xiaobo), and from electrochemical sensor (J. Liu et al.) to photocatalyst (Y. Zhang and S. Zhang et al.), as well as lithium ion batteries (Y. Jin et al.) and hydrogenation (Y. Zhang and N. Zheng et al.). It is exciting to report these contributions representing both traditional and modern research directions. We hope this issue will promote the 
development of the making technologies of low-dimensional inorganic materials and their further scaleup and potential applications in chemical engineering fields.

\section{Acknowledgments}

We would like to thank all the authors and coauthors who have made conspicuous contributions and all the reviewers for their valuable time and dedication to this special issue.

$$
\begin{array}{r}
\text { Lan Xiang } \\
\text { Guo Gao } \\
\text { Yunpeng Yin } \\
\text { Huijun Wu } \\
\text { Yongcheng Jin } \\
\text { Wancheng Zhu }
\end{array}
$$



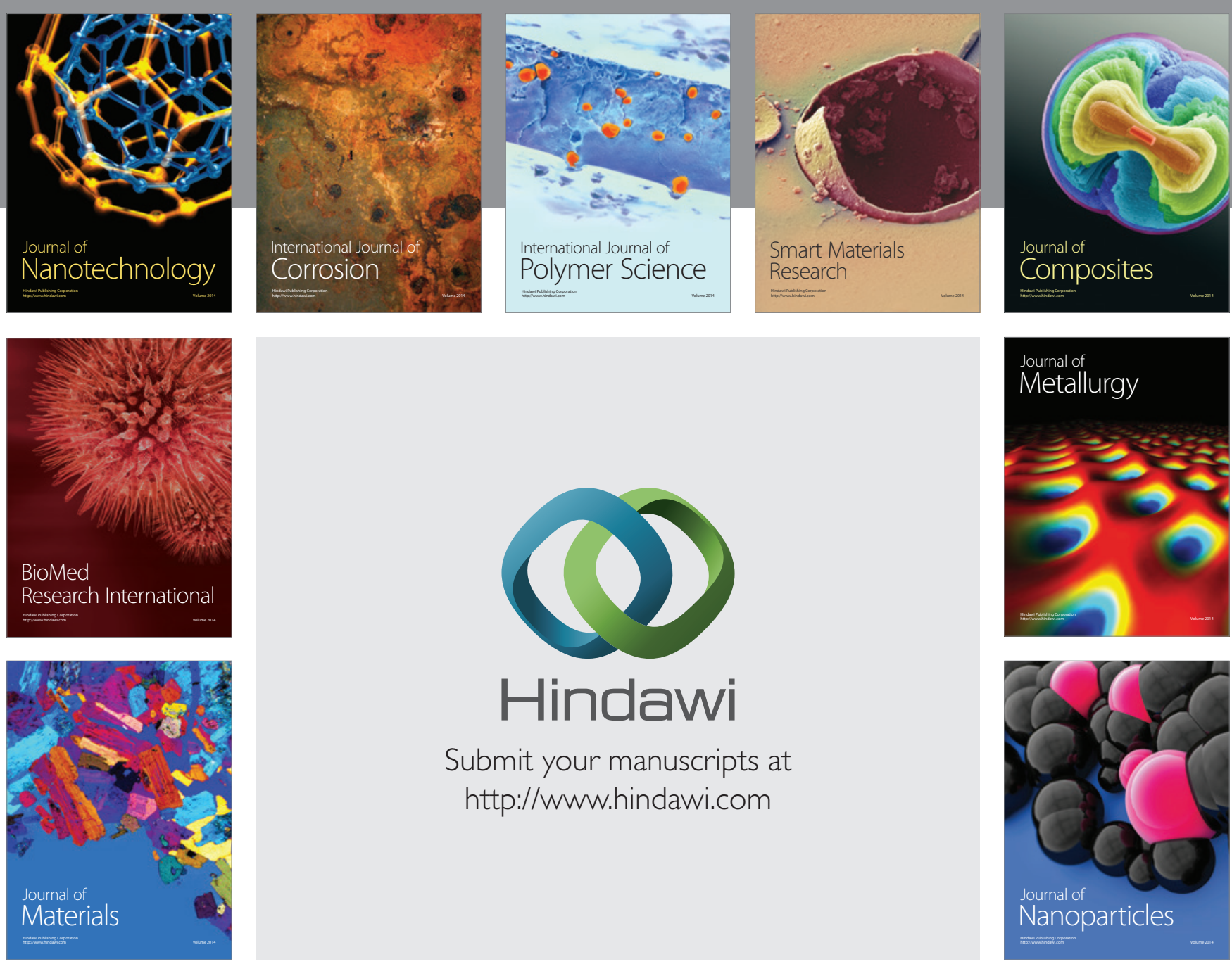

Submit your manuscripts at http://www.hindawi.com
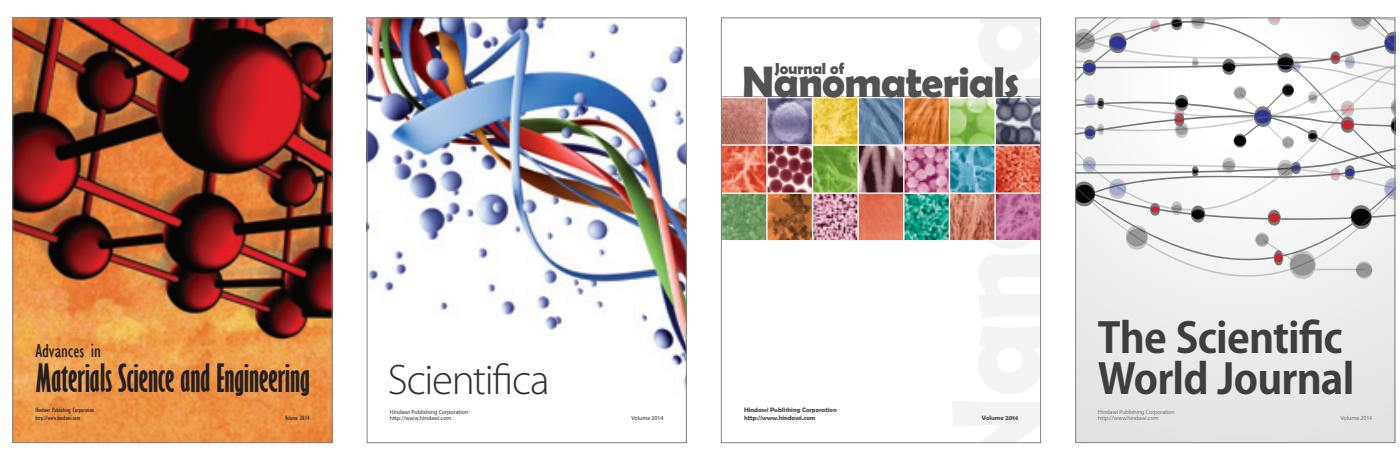

\section{The Scientific World Journal}
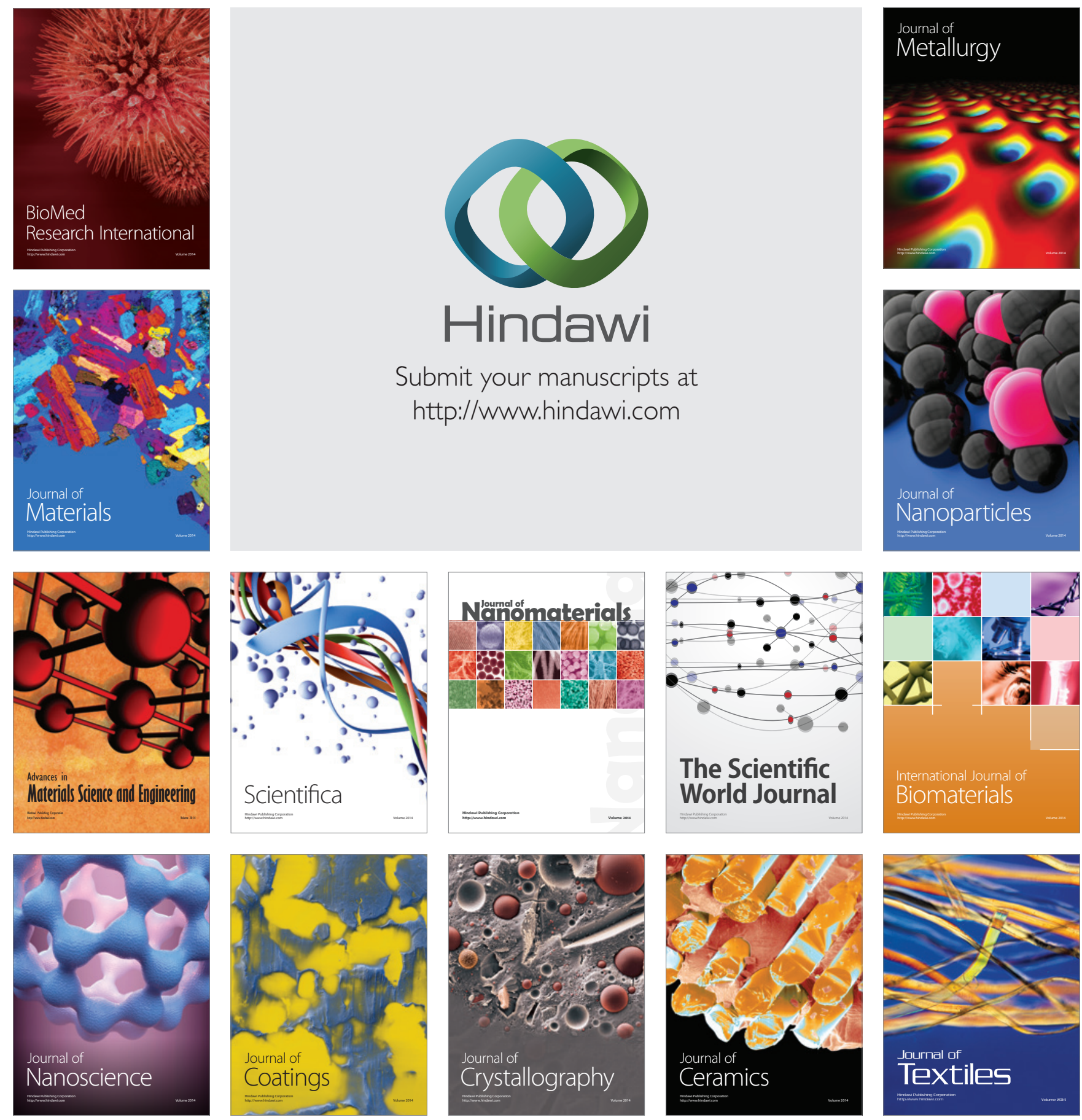